\title{
How a small virus changed our world and the special role of anosmia
}

In January 2020 we encountered the first news on a novel coronavirus (SARS-CoV-2) infecting the population of the Chinese city Wuhan and resulting for some patients in a potentially deadly pneumonia.

Currently, coronavirus disease 2019 (COVID-19) is spreading rapidly around the globe. For many years we have been warned that we would face a new pandemic, most likely with a zoonotic virus ${ }^{(1)}$. For COVID-19 it was shown that transmission can occur via droplets and aerosols that can remain in the air for some hours and also via contaminated surfaces. Extra risks have been suggested to exists for aerosol producing surgery in sinus- and skull-base surgery ${ }^{(2)}$. Ongoing transmission is especially difficult to prevent when the pathogen can be transmitted during the incubation period before the patient experiences symptoms. In March, Claire Hopkins and colleagues warned the rhinologic community and later health authorities about the significant increase in anosmia (and dysgeusia) related to (often further) asymptomatic COVID-19 and pointed to the possibility of recognizing COVID-19 patients by unexplained anosmia without other symptoms ${ }^{(3,4)}$. Today, Friday June 19th exactly 150 papers can be found in the literature on the relationship between COVID-19 and anosmia, of which a number are published in this issue of Rhinology. We are all aware that hyposmia and anosmia occur frequently in the (especially elderly) population and can be a sign of neurological disease ${ }^{(5,6)}$. Moreover, seasonality has been shown, independent of variation in viral infections ${ }^{(7)}$. Transient disorders of olfaction in upper respiratory tract infec- tions are frequent. Occasionally, persistent post-viral sensorineural olfactory dysfunction occurs, primarily caused by four families of viruses: rhinoviruses, influenza, coronaviruses, and parainfluenza ${ }^{(8)}$. However, in COVID-19, at least in some of the patients, anosmia/dysgeusia occurs early in the disease or as the presenting symptom. At present, it is still unclear whether COVID-19 represents a special case of viral infection attacking the olfactory system or that transient olfactory disfunction regularly occurs in other viral infections but is not reported as such. Future studies will be needed to determine whether this is indeed unique to SARS-Cov-2 rather than reflecting bias caused by our heightened interest.

For now, as has been advised both by European countries as the US a sudden anosmia/dysgeusia should be taken as a potential indication of SARS-CoV-2 infection and managed as such. Apart from the many COVID-19 publications that you can already find on our website, Rhinology Journal is bombarded by submitted papers of which a great selection is published in this issue. Although we are very happy with the large interest for the Journal we are forced to raise the bar of publication even further. To help more authors to publish their papers in our journal we started this year to accept letters to the editor as a format to ensure both rapid and constrained publications. For those who rather like to publish a longer original contribution that cannot be accepted because of page constrains we advise to publish in Rhinology online.

\section{References}

1. Barclay E. Predicting the next pandemic. Lancet, 2008. 372(9643): 1025-6

2. Van Gerven L, Hellings PW, Cox T, et al., Personal protection and delivery of rhinologic and endoscopic skull base procedures during the COVID-19 outbreak. Rhinology, 2020. 58(3): 289-294.

3. Gane SB, Kelly C, Hopkins C. Isolated sudden onset anosmia in COVID-19 infection. A novel syndrome? Rhinology, 2020. 58(3): 299-301.

4. Hopkins C, Surda P, Kumar N. Presentation of new onset anosmia during the COVID-19 pandemic Rhinology, 2020. 58(3): 295-298.

5. Foguem C, Lemdani M, Huart C. Parkinson disease in eldery patients: lessons from odour detection thresholds on olfacto-trigeminal interaction. Rhinology, 2018. 56(2): 127-132

6. Wang X, Zhang C, Xia X, Yang Y, Zhou C. Effect of gender on odor identification at different life stages: a meta-analysis. Rhinology, 2019. 57(5): 322-330.
7. Lotsch J, Hummel T. A data science-based analysis of seasonal patterns in outpatient presentations due to olfactory dysfunction. Rhinology, 2020;58(2):151-157.

8. Pellegrino R, Cooper KW, Di Pizio A., et al., Corona Viruses and the Chemical Senses: Past, Present, and Future. Chem Senses 2020;bjaa031. doi: 10.1093/chemse/bjaa031.

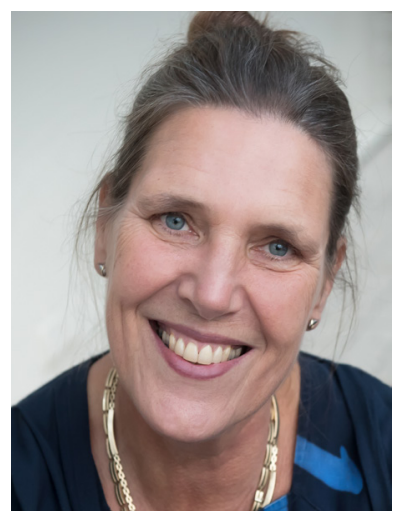

Wytske J. Fokkens, Editor-in Chief Amsterdam, the Netherlands 